\title{
Phonetic priming, neighborhood activation, and PARSYN
}

\author{
PAUL A. LUCE \\ State University of New York, Buffalo, New York \\ STEPHEN D. GOLDINGER \\ Arizona State University, Tempe, Arizona \\ EDWARD T. AUER, JR. \\ House Ear Institute, Los Angeles, California \\ and \\ MICHAEL S. VITEVITCH \\ Indiana University, Bloomington, Indiana
}

\begin{abstract}
Perceptual identification of spoken words in noise is less accurate when the target words are preceded by spoken phonetically related primes (Goldinger, Luce, \& Pisoni, 1989). The present investigation replicated and extended this finding. Subjects shadowed target words presented in the clear that were preceded by phonetically related or unrelated primes. In addition, primes were either higher or lower in frequency than the target words. Shadowing latencies were significantly longer for target words preceded by phonetically related primes, but only when the prime-target interstimulus interval was short (50 vs. $500 \mathrm{msec}$ ). These results demonstrate that phonetic priming does not depend on target degradation and that it affects processing time. We further demonstrated that PARSYN--a connectionist instantiation of the neighborhood activation model-accurately simulates the observed pattern of priming.
\end{abstract}

Virtually all current models of spoken word recognition share the assumption that the perception of spoken words involves two fundamental processes: activation and competition (see P. A. Luce \& Pisoni, 1998; Marslen-Wilson, 1989; McClelland \& Elman, 1986; Norris, 1994). In such activation-competition models, the hallmark of the discrimination process is competition among multiple representations of words activated in memory. As a result, the role of competition has been a primary focus of research and theory on spoken word recognition in the last few years (e.g., Cluff \& Luce, 1990; Goldinger, Luce, \& Pisoni, 1989; Marslen-Wilson, 1989; McQueen, Norris, \& Cutler, 1994; Norris, McQueen, \& Cutler, 1995; Vitevitch \& Luce, 1998, 1999).

One example of an activation-competition model is the neighborhood activation model (NAM; P. A. Luce \& Pisoni, 1998). According to NAM, stimulus input activates a set (or neighborhood) of acoustic-phonetic patterns in

This research was supported in part by Research Grants R01 DC 0265801-A2 and R29 DC 02629-03 from the National Institute on Deafness and Other Communication Disorders. National Institutes of Health. We thank Dennis Norris and an anonymous reviewer for their advice and comments. We also thank Jim Sawusch for many helpful discussions and Michael S. Cluff for his assistance in running subjects. Correspondence concerning this article should be addressed to $\mathrm{P}$. A. Luce, Department of Psychology, State University of New York, Buffalo, NY 14260 (e-mail: paul@deuro.fss.buffalo.edu). memory. Patterns are activated to the degree to which they match the stimulus input (see Marslen-Wilson, 1989, Morton, 1969, and Norris, 1994, for similar proposals). These acoustic-phonetic patterns then activate a system of word decision units that are tuned to the patterns. Throughout the recognition process, the word decision units monitor three sources of information: (1) the activation levels of the acoustic-phonetic patterns to which the units are tuned, (2) higher level lexical information (specifically, lexical frequency), and (3) the overall level of activity in the entire system of decision units. It is assumed that each of the decision units continuously computes decision values on the basis of these three sources of information.

Decision values are computed using a frequency-biased, activation-based version of R. D. Luce's (1959) choice rule (see P. A. Luce, 1986, for a complete discussion of the choice rule; see also McClelland \& Elman, 1986). In particular, the activation level of a pattern is continuously compared with the overall activity level within the system. When the activity level is high (i.e., when many patterns, or neighbors, are consistent with the input), the decision values computed by the word units will tend to be low. Furthermore, lexical frequency biases the decisions of the word units by differentially amplifying activation levels of patterns corresponding to high-and low-frequency words. All other things being equal, decision values for high-frequency words will exceed those for low-frequency 
words. Recognition of a given word is accomplished when the decision value computed by a single word decision unit surpasses criterion (see Marslen-Wilson, 1989, and McClelland \& Elman, 1986, for similar accounts of the recognition process).

NAM makes several predictions regarding the effects of neighborhood and frequency effects on spoken word recognition. First, if stimulus input activates a relatively large number of similar acoustic-phonetic patterns in memory, word recognition is predicted to be slower and less accurate. That is, words with many similar sounding neighbors should be responded to less quickly and accurately than words with few similar sounding neighbors. Second, NAM predicts that the frequency of the neighborhood should affect recognition. In particular, the model predicts that, all things being equal, words with highfrequency neighbors should be responded to less quickly and accurately than words with low-frequency neighbors. Finally, NAM predicts processing advantages for highfrequency words (although the frequency effect should be mediated by neighborhood characteristics).

There is now considerable empirical support for each of these predictions (Cluff \& Luce, 1990; Goldinger et al., 1989; P. A. Luce, 1986; P. A. Luce \& Pisoni, 1998; P. A. Luce, Pisoni, \& Goldinger, 1990; Vitevitch \& Luce, 1998 , 1999; see also Goldinger, Luce, Pisoni, \& Marcario, 1992). These studies have demonstrated that spoken word recognition is influenced by the number and nature of items activated in memory by stimulus input. Some of the strongest evidence for NAM (and, by implication, other activation-competition models) was provided by Goldinger et al. (1989), the first form-based priming experiment to demonstrate inhibitory effects in spoken word recognition. In this study, target words were preceded by phonetic neighbors (related primes) or by phonetically unrelated words (unrelated primes). Examples of related prime-target pairs are VEER-BULL and PAR-TALL. The primes (which were either high-or low-frequency words) were presented in the clear, and the targets were presented in noise. In addition, the primes and the targets were separated by either a 50 - or a 500 -msec interstimulus interval (ISI). The subjects' task was to identify the target.

On the basis of NAM, Goldinger et al. (1989) predicted that a phonetically related prime should raise the activity level of the neighborhood of the target, thus lowering the probability of correct identification. That is, residual activation from the related prime should increase competition in the neighborhood of the target word, thus producing inhibition priming. (We use the term inhibition priming to denote decrements in performance as a function of the relatedness of the prime.) Their results confirmed this prediction: Phonetically related primes inhibited target identification, but only when the ISI between the primes and targets was short $(50 \mathrm{msec})$. When the ISI was increased to $500 \mathrm{msec}$, no inhibition was observed, suggesting that the priming was not strategic. More re- cently, Goldinger et al. (1992) demonstrated that phonetic priming is not affected by manipulation of subject strategies, whereas other types of form-based priming appear to have strategic components (e.g., segment repetition; see Monsell \& Hirsh, 1998; Slowiaczek, Nusbaum, \& Pisoni, 1987).

Goldinger et al.'s initial demonstration of inhibition priming in spoken word recognition has important theoretical implications. In particular, models of recognition such as Shortlist (McQueen et al., 1994; Norris, McQueen, \& Cutler, 1995) and NAM depend crucially on the empirical demonstration of such competition effects. However, there are several shortcomings in the current work on phonetic priming. First, the effect has only been demonstrated in tasks using targets degraded by noise. Although we can be fairly certain that stimulus degradation does not induce strategies that may produce the effect, we have no direct evidence that phonetic priming is present for targets in the clear (see Goldinger et al., 1992). Even if the effect is not strategy based, it may depend on incomplete or poorly specificd target information, which makes generalization to fluent perception somewhat tenuous. Another important shortcoming of the Goldinger et al. (1989) study is that there is no indication that phonetic priming affects processing time (as predicted by activation-competition models). Some researchers (e.g., Radeau, Morais, \& Dewier, 1989) have argued that adequate tests of theories of spoken word recognition should employ reaction time (RT) paradigms that minimize postperceptual processing induced either by stimulus degradation (as in perceptual identification) or by task demands (as in lexical decision). Finally, Goldinger et al. (1989) made no attempt to simulate the phonetic priming effect. Although NAM makes qualitative mathematical predictions of inhibition priming (see Goldinger et al., 1989; P. A. Luce, 1986), simulating the effect in a formal modeling architecture would provide further support for our interpretation of the locus of the effect.

The present study was aimed at addressing these shortcomings. We used a primed shadowing task (Radeau et al., 1989) in which subjects hear a prime and a target, both presented in the clear at a comfortable listening level, and must repeat back the target. The time between the onset of the target and the onset of the shadowing response is measured. This task enables us to examine the effects of phonetic priming on processing times, using stimuli that are not made purposefully difficult to perceive. Use of degraded, poorly specified stimuli in the perceptual identification task may have the undesirable consequence of encouraging subjects to employ the prime in generating a response, thereby calling into question any activation-based account of possible priming effects. In addition to using the naming task, we attempted to simulate the effects of inhibition priming in a connectionist model of spoken word recognition - dubbed PARSYNdeveloped by Auer and Luce (2000). This model is essen- 
tially a processing instantiation of NAM and provides a mechanistic framework for simulating possible formbased priming effects.

\section{EXPERIMENT 1}

\begin{abstract}
Method
Subjects. Twenty-four undergraduates at the State University of New York at Buffalo participated in partial fulfillment of requirements for an introductory psychology course. All the subjects were native English speakers and reported no history of speech or hearing disorders.
\end{abstract}

Stimuli. Ninety-two pairs of phonetically related consonantvowel-consonant (CVC) prime-target pairs were selected using a computerized lexicon based on Webster's pocket dictionary (1967). In addition, unrelated CVC primes were selected for each of the 92 targets. All the primes and targets had a rated familiarity of 6 or above on a 7-point scale $(1=$ don't know the word, $7=$ know' the word and its meaning; Nusbaum, Pisoni, \& Davis, 1984). Examples of the primes and the targets are shown in Table 1, and summary statistics for the stimuli are shown in Table 2. All stimuli are listed in the Appendix.

The related prime-target pairs were created by searching the database for the nearest neighbor of each target that had no positional phoneme overlap. To select the neighbors, we used a database of subjective similarity ratings for all initial consonants, vowels, and final consonants. Twenty-four subjects judged the similarity of speech sounds in three separate conditions. In the first condition, the subjects judged the similarity of each prevocalic consonant paired with all the other prevocalic consonants. In the second condition, the subjects judged the similarity of each postvocalic consonant paired with all the other postvocalic consonants. In the third condition, the subjects judged each vowel paired with all the other vowels. The subjects were asked to rate the similarity of the pairs of consonants or vowels. For example, in the prevocalic condition, the subjects were presented with pairs of different $C V$ tokens and were asked to rate how similar the two consonants sounded. Subjective ratings ranged from 1 , indicating maximal dissimilarity, to 10 , indicating maximal similarity. Eight subjects in each condition made five judgments on each of the possible combinations of tokens for that condition.

The ratings were transformed to values representing the degree of similarity among the initial consonants, vowels, and final consonants. We then used these values to choose phonetically related primes and targets from the lexicon. Words composed of segments judged to be similar to the segments of the targets were selected as related primes. For example, /fon / (fawn) was chosen as a prime for $/ \theta \wedge \mathrm{m} /$ (thumb) because, based on the subjective similarity judgments, $/ \mathrm{f} /$ was judged to be similar to $/ \theta /, / \mathrm{o} /$ was judged similar to $/ \mathrm{A} /$, and $/ \mathbf{n} /$ was judged similar to $/ \mathrm{m} /$. The unrelated primes were composed of segments that were not judged similar to the segments of the targets. In order to compute a similarity score for each prime-target pair, we multiplied the transformed similarity ratings for each of the three

Table 1

Examples of Stimuli for Experiments 1 and 2

\begin{tabular}{lll}
\hline Target & Related Prime & Unrelated Prime \\
\hline rule & war $(\mathrm{H})$ & deep \\
veil & fair $(\mathrm{H})$ & chose \\
van & thing $(\mathrm{H})$ & sake \\
shed & sage $(\mathrm{L})$ & coil \\
coin & tomb $(\mathrm{L})$ & leech \\
thumb & fawn $(\mathrm{L})$ & cheat \\
\hline
\end{tabular}

Note-Prime frequency $(\mathrm{H}=$ high, $\mathrm{L}=$ low $)$ is indicated in parentheses. segments in question. The average phonetic similarity scores based on the products of the transformed segmental similarity ratings are shown in Table 2 for both the related and the unrelated prime-target pairs.

Half of the targets were paired with related and unrelated primes that were higher in frequency than the targets. The other half of the targets were paired with related and unrelated primes that were lower in frequency. High-frequency primes had a mean log frequency of 2.98 (Kučera \& Francis, 1967); low-frequency primes had a mean $\log$ frequency of 1.66 . Targets had an overall mean $\log$ frequency of 2.23. Mean $\log$ frequency for targets that followed highfrequency primes was 2.16 ; mean $\log$ frequency for targets that followed low-frequency primes was 2.30 (see Table 2 ). This difference was not significant $[F(1,90)=3.21, p>.05]$.

Targets that followed high-frequency primes were also equated with targets that followed low-frequency primes on log-frequencyweighted neighborhood density (see Table 2). Neighborhoods were computed by using the on-line database and the transformed segmental similarity-scaling data. Neighborhood density values for targets that followed high- and low-frequency primes were not significantly different [high density $=.34$; low density $=.33 ; F(1,90)<$ 1.0]. In addition, high-frequency and low-frequency primes were equated on their degree of similarity to the targets, again using the segmental similarity data. High- and low-frequency related primes were equally similar to the targets [high frequency $=.024$; low frequency $=.025 ; F(1,90)<1.0]$. Likewise, high- and low-frequency unrelated primes were equally dissimilar to the targets [high frequency $=.006$; low frequency $=.006 ; F(1,90)<1.0]$.

Because every target had two corresponding primes (related and unrelated), two lists of stimuli were constructed to ensure that no subject heard a given target more than once. Targets preceded by related primes in List 1 were preceded by unrelated primes in List 2. Likewise, targets preceded by related primes in List 2 were preceded by unrelated primes in List 1 . Thus, every target served as its own control. A given list was presented to only one group of subjects. The stimuli were recorded by the same male talker of a Midwestern dialect who produced the stimuli for the segmental similarityrating study. All the words were spoken in isolation, low-pass filtered at $4.8 \mathrm{kHz}$, and digitized at a sampling rate of $10 \mathrm{kHz}$, using a $12-$ bit analogue-to-digital converter. All the words were edited into individual files and stored on computer disk.

Design. Two levels of two variables were examined: (1) prime type (related vs. unrelated) and (2) prime frequency (high vs. low). The dependent measures were shadowing latency and percentage of words correctly repeated.

Procedure. The subjects were tested individually in a booth equipped with a voice response key interfaced to a minicomputer that controlled stimulus presentation and response collection. On each trial, the subjects heard a prime and a target presented over headphones at a comfortable listening level. The prime and the target were separated by a 50 -msec ISI. The subjects were instructed to repeat back the second word of the pair as quickly and as accurately as possible into a microphone attached to the headphones. The subjects were not required to make a response to the prime. RTs were recorded from the onset of the target word to the onset of the shadowing response. Accuracy was monitored by the experimenter, who was seated next to the subject. Each subject received five practice trials followed by a randomly ordered presentation of 92 primetarget pairs.

\section{Results and Discussion}

Shadowing RTs and percentages correct are shown in Table 3. Means for targets preceded by related and unrelated primes are shown in the first and second rows, respectively. Means for targets preceded by high- and low- 
Table 2

Statistics for Targets and Primes

\begin{tabular}{lccc}
\hline \multicolumn{1}{c}{ Stimuli } & & $\begin{array}{c}\text { Frequency-Weighted } \\
\text { Leighborhood Density }\end{array}$ & $\begin{array}{c}\text { Phonetic } \\
\text { Similarity Score }\end{array}$ \\
\hline Targets & Log Frequency & & \\
$\quad$ Following high-frequency primes & 2.16 & .34 & \\
$\quad$ Following low-frequency primes & 2.30 & .33 & \\
Primes & & & .024 \\
$\quad$ Related & 3.02 & .025 \\
$\quad$ High frequency & 1.53 & & .006 \\
$\quad$ Low frequency & & .006 \\
$\quad$ Unrelated & 2.94 & \\
$\quad$ High frequency & 1.79 & \\
$\quad$ Low frequency & & & \\
\hline
\end{tabular}

frequency primes are shown in the columns. Differences between RTs to related and unrelated primes are shown in the third row. Positive differences indicate inhibition priming. Two items were deleted from all subsequent analyses because average RTs for those items were over two standard deviations above the mean for all items.

A two-way analysis of variance (ANOVA; prime type $X$ prime frequency) was performed on the mean latencies and percentages correct for both subjects $\left(F_{\mathrm{s}}\right)$ and items $\left(F_{1}\right)$. For the RT data, we obtained significant effects of prime type $\left[F_{\mathrm{s}}(1,23)=8.50, p<.01\right]$ and prime frequency $\left[F_{\mathrm{s}}(1,23)=16.56, p<.001\right]$, although only the effect of prime type was significant in the items analysis [prime type: $F_{\mathrm{i}}(1,88)=5.49, p<.03 ;$ prime frequency: $\left.F_{\mathrm{i}}(1,88)<1.0\right]$. The prime type $\times$ prime frequency interaction was not significant by subjects or items $\left[F_{\mathrm{s}}(1,23)<\right.$ 1.0 and $\left.F_{\mathrm{i}}(1,88)<1.0\right]$. Targets preceded by high-frequency related primes were responded to $11 \mathrm{msec}$ slower than targets preceded by high-frequency unrelated primes. Targets preceded by low-frequency related primes were responded to $26 \mathrm{msec}$ slower than targets preceded by lowfrequency unrelated primes. No effects for accuracy were significant at the .05 alpha level.

Experiment 1 replicated the previous work demonstrating form-based inhibition priming in spoken word recognition (Goldinger et al., 1989; Goldinger et al., 1992). It also extended prior work by demonstrating (1) that the effect does not depend on degrading the targets with noise and (2) that phonetically related primes have demonstrable effects on processing times for targets. Finally, although the prime type $\times$ prime frequency interaction was not significant, the pattern of results paralleled Goldinger et al.'s (1989) original finding that low-frequency primes produce more inhibition than do high-frequency primes.

In order to confirm that the inhibition priming effects in Experiment 1 were due solely (or, at least, primarily) to competition at the level of phonetic pattern activation, we examined the time course of the effect by introducing a 500-msec ISI between the primes and the targets. It is typically assumed that once a word is recognized, its activation level returns to resting level (Collins \& Loftus, 1975; Goldinger et al., 1989; McClelland \& Elman, 1986). Thus, according to an activation-based account of inhibition priming, the activation of the prime should fade over the 500 -msec interval, leaving little or no residual activation to interfere with the processing of the target.

\section{EXPERIMENT 2}

\section{Method}

The stimuli, experimental design, and procedure were identical to those in Experiment 1. The only difference was the 500 -msec ISI between the prime and the target.

Subjects. Twenty-four undergraduates at the State University of New York at Buffalo participated in partial fulfillment of requirements for an introductory psychology course. None of the subjects in Experiment 2 participated in Experiment 1. All the subjects were native English speakers and reported no history of speech or hearing disorders.

Table 3

Reaction Time (RT, in Milliseconds), Percentage Correct (PC), and Standard Error for Target Items Following High- and Low-Frequency Related and Unrelated Primes for the 50-Msec Interstimulus Interval Condition

\begin{tabular}{|c|c|c|c|c|c|c|c|c|}
\hline \multirow[b]{4}{*}{ Prime Type } & \multicolumn{8}{|c|}{ Prime Frequency } \\
\hline & \multicolumn{4}{|c|}{ High } & \multicolumn{4}{|c|}{ Low } \\
\hline & \multicolumn{2}{|c|}{ RT } & \multicolumn{2}{|c|}{$\mathrm{PC}$} & \multicolumn{2}{|c|}{ RT } & \multicolumn{2}{|c|}{$\mathrm{PC}$} \\
\hline & $M$ & $S E$ & $M$ & $S E$ & $M$ & $S E$ & $M$ & $S E$ \\
\hline Related & 780 & 18 & 94 & .01 & 805 & 19 & 91 & .01 \\
\hline Unrelated & 805 & 19 & 91 & .01 & 779 & 16 & 92 & .01 \\
\hline Difference in RT & 11 & & & & 26 & & & \\
\hline
\end{tabular}

Note-Positive values for "Difference in RT" indicate inhibition priming. 
Table 4

Reaction Time (RT, in Milliseconds), Percentage Correct (PC), and Standard Error for Target Items Following High- and Low-Frequency Related and Unrelated Primes for the 500-Msec Interstimulus Interval Condition

\begin{tabular}{|c|c|c|c|c|c|c|c|c|}
\hline \multirow[b]{4}{*}{ Prime Type } & \multicolumn{8}{|c|}{ Prime Frequency } \\
\hline & \multicolumn{4}{|c|}{ High } & \multicolumn{4}{|c|}{ Low } \\
\hline & \multicolumn{2}{|c|}{ RT } & \multicolumn{2}{|c|}{$\mathrm{PC}$} & \multicolumn{2}{|c|}{ RT } & \multicolumn{2}{|c|}{$\mathrm{PC}$} \\
\hline & $M$ & $S E$ & $M$ & $S E$ & $M$ & $S E$ & $M$ & $S E$ \\
\hline Related & 806 & 29 & 95 & .01 & 819 & 28 & 89 & .02 \\
\hline Unrelated & 802 & 28 & 95 & .01 & 821 & 32 & 92 & .02 \\
\hline Difference in RT & 4 & & & & -3 & & & \\
\hline
\end{tabular}

Note-Positive values for "Difference in RT" indicate inhibition priming.

\section{Results and Discussion}

Shadowing RTs and percentages correct are shown in Table 4. Means for targets preceded by related and unrelated primes are shown in the first and second rows, respectively. Means for targets preceded by high- and lowfrequency primes are shown in the columns. Differences between RTs to related and unrelated primes are shown in the third row. Positive differences indicate inhibition priming. Three subjects were deleted because their average RTs were over two standard deviations above the mean RT for all the subjects. In addition, five items were deleted because their average RTs were over two standard deviations above the mean for all items. A two-way ANOVA (prime type $\times$ prime frequency) was performed on the mean latencies and percentages correct. For the RT data, we obtained no effect of prime type in either the subjects or the items analysis $\left[F_{\mathrm{s}}(1,20)<1.0 ; F_{\mathrm{i}}(1,85)<\right.$ 1.0]. A significant effect of prime frequency was obtained in the subjects analysis $\left[F_{\mathrm{s}}(1,20)=8.83, p<.01\right]$, but not in the items analysis $\left[F_{\mathrm{i}}(1,85)<1.0\right]$. For the accuracy data, there was again no effect of prime type $\left[F_{\mathrm{s}}(1,20)<\right.$ $\left.1.0 ; F_{\mathrm{i}}(1,85)<1.0\right]$. However, as for the RT data, a significant effect of prime frequency was obtained in the subjects analysis $\left[F_{\mathrm{s}}(1,20)=11.62, p<.003\right]$, but not in the items analysis $\left[F_{\mathrm{i}}(1,85)=2.51, p>.10\right]$.

As was predicted, increasing the ISI between the prime and the target eliminated the effect of inhibition priming. When the data of Experiments 1 and 2 were combined, the prime type $\times$ ISI interaction was significant by subjects $\left[F_{\mathrm{s}}(1,43)=4.63, p<.04\right]$ and marginally significant by items $\left[F_{\mathrm{i}}(2,173)=3.04, p=.08\right]$. Apparently, the residual activation of the prime is short-lived, fading over the longer interval. Although there was again some evidence for an overall effect of prime frequency (independent of phonetic relatedness), the effect failed to reach significance in the items analysis.

\section{SIMULATION}

To further explore the locus of the observed inhibition effects, we simulated the pattern of results, using a connectionist model of spoken word recognition, PARSYN (Auer \& Luce, 2000). We chose PARSYN primarily because the model directly encodes in its activation levels the subjective similarity measures used to determine pho- netic similarity in the present set of experiments. We believe that a number of current connectionist models-in particular, Shortlist and TRACE-make predictions regarding phonetic priming that are virtually identical to those of PARSYN. Thus, we take the behavior of PARSYN to be indicative of a general class of activationcompetition models. Any success or failure of this particular model should generalize to any connectionist model with similar architectures and processing schemes.

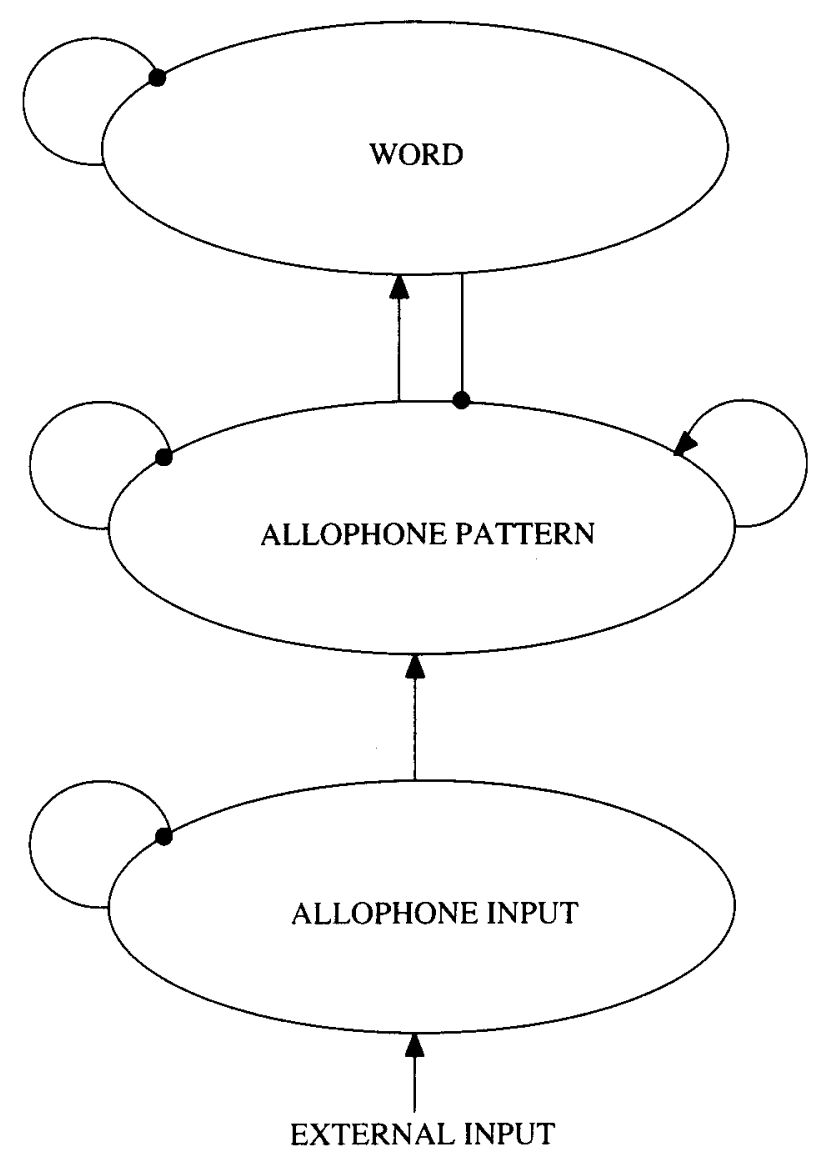

Figure 1. The PARSYN model of spoken word recognition. Facilitative connections are depicted by lines terminating in arrows, and inhibitory connections are depicted by lines terminating in filled circles. 
PARSYN is a processing instantiation of NAM that has three levels of units: (1) an input level, (2) a pattern level, and (3) a word level (see Figure 1). Connections between units within a level are mutually inhibitory, with one exception: Links among allophones at the pattern level are facilitative across temporal positions (see below). Connections between levels are facilitative, also with one exception: The word level sends inhibitory information back to the pattern level, essentially quelling activation in the system once a single word has gained a marked advantage over its competitors. (Although this last feature of PARSYN contrasts with Shortlist's autonomous segmental and lexical levels, there is no reason to believe that this difference should produce marked differences in predictions for the two models.)

Each layer consists of several interconnected units. The activation level of a unit represents the strength of the hypothesis that a given segment or word has been presented to the system. The first, or input, layer consists of position-specific allophonic units arranged into banks of receptors corresponding to the temporal sequence of the input (for empirical support for the use of positionspecific allophonic units, see Gagnon, Palmer, \& Sawusch, 2000). In its current form, PARSYN is capable of processing input consisting of four segments, where the fourth segment is a word boundary marker. Thus, the input layer consists of banks of receptors corresponding to four temporal positions. In the first temporal position, there are 38 allophones corresponding to the vowels and prevocalic consonants. In the next three temporal positions, there are duplicate sets of 50 nodes corresponding to prevocalic consonants, vowels, postvocalic consonants, and a word boundary marker. (Because postvocalic consonants and the word boundary marker cannot occur in initial position, the number of nodes is larger in the noninitial positions.) The units in the input layer receive facilitative input from an external input vector. Within a temporal position, all of the units are mutually inhibitory.

The second, or pattern, layer of units exactly duplicates the input layer, with units at the pattern level receiving direct facilitative input from the allophone input units. However, the input and pattern layers differ in the interconnections between the units. Whereas banks of units at the input level do not directly interact over time, units at the pattern level receive facilitative input from other pattern layer units in preceding and/or following temporal positions. The weights on these within-level connections correspond to log-frequency-weighted forward and backward position-specific transitional probabilities. These transitional probabilities were computed using the 20,000 word Webster's on-line lexical database. These transitional probabilities are assumed to represent the (first-order) probabilistic phonotactic constraints of the words in English. In addition, the resting levels of the nodes in the pattern layer correspond to the log-frequency-weighted position-specific probabilities of occurrence, also computed using Webster's on-line lexical database. Thus, allophones that commonly occur in a given temporal position will begin processing with higher resting activation levels than will less commonly occurring allophones. In the pattern layer, all of the units within a temporal position are capable of mutual inhibition.

The third, or word, layer consists of 8,873 word units. The words range from 1 to 15 phonemes in length and have a rated familiarity of 6 or greater on a 7-point familiarity scale (Nusbaum et al., 1984). Word level units receive facilitative input from their constituent positionspecific allophones at the pattern level. Each word level unit is capable of inhibiting all the other word units.

\section{Input to the Model}

A position-specific allophonic transcription of the input word is translated into a sequence of input vectors that correspond to the cycle of processing and the temporal position of the input. Units at the allophone input layer are then activated as a function of the similarity of the allophones to the input. Input to the system is staged in such a way that the input vector corresponding to an input allophone is presented over several processing cycles. In naturally produced speech, the durations of allophones in all positions are, of course, not equal. In order to approximate these differences in duration, gross adjustments were made in the number of cycles of input for a given position. Vowel information was presented for the longest duration, followed by consonantal information. Word boundary information was presented for the shortest duration. Furthermore, in order to "co-articulate" the input vectors, the presentation of the vectors corresponding to initial consonants and vowels overlapped by two processing cycles, vowels and final consonants overlapped by four processing cycles, and the final consonant and the word boundary information overlapped by two processing cycles.

Activation values for the input vector are computed from the subjective similarity scores for each initial consonant, vowel, and final consonant (see Experiment 1). By way of example, consider computation of the activation values for initial consonants: First, the similarity scores for initial consonant $i$ compared with all of the $j$ initial consonants are summed $\left(\sum S_{i j}\right)$. The activation values corresponding to each initial consonant $j$, given the initial consonant $i$, are then computed by dividing the similarity score for initial consonants $i$ and $j$ by the sum of all similarity scores:

$$
a_{i j}=\frac{S_{i j}}{\sum S_{i j}} .
$$

where $a_{l_{i}}$ is the activation level of the $i$ th allophone at the input level, $I$. This process creates an input vector containing activity values corresponding to all the initial consonants, given the initial consonant $i$ as input. Activation val- 
ues for allophones in each temporal position are computed in a similar manner.

\section{Processing Dynamics}

The processing assumptions of the model are similar to those of McClelland and Elman's (1986) TRACE model and McClelland and Rumelhart's (1981) interactive activation model. Activation values of units are restricted to a range from -.3 to 1.0. At each processing cycle, all the units in the system are updated in a parallel manner. For each unit at each processing cycle, a net input value is computed on the basis of the activity of all of its connected units. The net input, or net $t_{i}$, to unit $i$ is given by $n e t_{i}=f a c_{i}+i_{n h i} b_{i}$, where $f a c$ represents facilitation and inhib represents inhibition. The facilitation and inhibition into a node are computed on the basis of the type of connection, the activation level of the unit from which the activation originates, and the weight on the connection between the two nodes. Facilitation and inhibition for unit $i$ from unit $j$ are computed by

$$
\begin{aligned}
& \text { If } a_{j}>0.0, \\
& f a c_{i}=\sum a_{j} w_{i j}, \text { where } w_{i j}>0.0, \text { and } \\
& \text { inhib } b_{i j}=\sum a_{j} w_{i j}, \text { where } w_{i j}<0.0,
\end{aligned}
$$

where $f a c_{i}$ is the facilitation and inhib $b_{i}$ the inhibition impinging on unit $i, a_{j}$ represents the activation level of unit $j$, and $w_{i j}$ is the weight on the connection between unit $i$ and unit $j$. The amount of change in activation for unit $i$ is then computed on the basis of its net input, current activation value, and the decay rate. The change in activation is given by

$$
\begin{aligned}
& \text { If } n e t_{i}>0.0, \\
& \Delta a_{i}=\left(\max -a_{i}\right) n e t_{i}-\operatorname{decay}\left(a_{i}\right) ; \\
& \text { If } \text { net }_{i} \leq 0.0, \\
& \Delta a_{i}=\left(a_{i}-\min \right) \text { net }_{i}-\operatorname{decay}\left(a_{i}\right),
\end{aligned}
$$

where $\Delta a_{i}$ represents the change in activation of unit $i, a_{i}$; represents the activation of unit $i$, max represents the maximum activation a unit may reach, $\min$ represents the minimum activation value a unit may reach, net $_{i}$ represents the net input to a unit $i$, and decay represents the rate at which activations return to the resting value of zero.

Table 5

Parameter Settings for the PARSYN Simulation

\begin{tabular}{llc}
\hline \multicolumn{1}{c}{ Parameter } & \multicolumn{1}{c}{ Level } & Setting \\
\hline Decay rate & Input & 0.100 \\
& Pattern & 0.100 \\
& Word & 0.200 \\
Within-level inhibition & Input & 0.050 \\
& Pattern & 0.050 \\
Between-level inhibition & Word & 0.050 \\
Between-level facilitation & Word to pattern & 0.001 \\
& Input to pattern & 0.050 \\
& Pattern to word & 0.050 \\
\hline
\end{tabular}

\section{Parameters}

The nine relevant parameters for this simulation are shown in Table 5 . The first three parameters set the decay rate for nodes at each of the levels. The decay rate controls how rapidly an active unit will return to its resting level of activation. When set to high values, these parameters will quickly reduce the number of units that remain active when there is little or no facilitative input to the unit. Conversely, when the decay parameters are set to low values, units may remain active even when there is little facilitative input. The next three parameters set the amount of mutual inhibition within each level. Mutual inhibition serves to enhance the contrast between differentially activated units. Inhibition also allows for competition between activated units. The final three parameters control the degree of between-level inhibition and facilitation.

\section{Output}

Activations are transformed and scaled to give weight to stronger activations and to ensure all positive values. Also, in order to encode word frequency in the model, a frequency-weighted version of the activation transformation function was implemented to represent the activation levels of the word nodes at the lexical level. In this equation, the log-transformed Kučera and Francis (1967) frequency of a word was multiplied by the transformed activation of that word: $a c t=e^{k a_{i} * \log (f r e q) \text {, where } a c t}$ represents the transformed activation, $k$ represents a scaling constant, $a_{i}$ represents the activation level of the unit, and freq represents the transformed Kučera and Francis frequency of the word for which the unit stands. In order to avoid zero values, words without Kučera and Francis frequencies were assigned a frequency of 1 . Furthermore, all the frequencies were multiplied by a constant to avoid zero values resulting from the log transform. For the present simulation, the output of the model is obtained from the lexical level. The probability of choosing a particular alternative for a response at the lexical level is computed by applying R. D. Luce's (1959) choice rule to the exponentially transformed set of activations:

$$
P(\text { target })=\frac{a(\text { target })}{a(\text { target })+\sum\left[a\left(\text { competitor }_{j}\right)\right]},
$$

where $P$ (target) represents the probability of choosing the target word, $a$ (target) represents the transformed activation level of the target word unit, and $\sum\left[a\left(\right.\right.$ competitor $\left.\left._{j}\right)\right]$ represents the total transformed activation levels of word units other than the target. The output of the model is a function of the $P$ (target) value at the final processing cycle of the input item. High values correspond to words that are predicted by the model to be processed more rapidly.

\section{Simulation of Phonetic Priming}

In order to simulate the present pattern of results, we presented the 92 prime-target pairs to the model. We first presented a related or unrelated prime and allowed the 
Table 6

Output Values From PARSYN for Target Items Following High- and Low-Frequency Related and Unrelated Primes

\begin{tabular}{cccc}
\hline \multirow{2}{*}{ Prime Frequency } & \multicolumn{2}{c}{ Prime Type } & \\
\cline { 2 - 3 } & Related & Unrelated & Difference \\
\hline High & .19 & .21 & -.02 \\
Low & .17 & .22 & -.05 \\
\hline
\end{tabular}

Note-Negative values under the column labeled "Difference" indicate inhibition priming.

model to process the prime. We then presented the target and monitored the output of the word level unit corresponding to that item. Again, output values correspond to relative activation levels for an item: the higher the output value, the higher the activation level.

The model successfully recognized all but two targets (one in each of the low-frequency prime conditions). These two items were excluded from the subsequent analysis. The results of the simulation are shown in Table 6. Output values for target words following related and unrelated primes are shown in the left and right columns, respectively. Output values for target words following highand low-frequency primes are shown in the upper and lower rows, respectively. Item analyses revealed significantly lower output values for targets following related primes, as compared with output values for targets following unrelated primes $[F(1,89)=4.624, p<.05]$. (The main effect of prime frequency and the interaction of prime frequency and prime type were not significant; both $F \mathrm{~s}<1$.) Thus, the model produced inhibition priming (i.e., lower activation levels for target words preceded by related primes than for those preceded by unrelated primes). Note that, as in Experiments 1 and 2, the same targets were presented in both the related and the unrelated priming conditions. Therefore, the differences in output values from the model can only be attributed to the effects of the preceding prime.

PARSYN accurately simulates the pattern of inhibition priming observed both in Experiment 1 of the present study and in Goldinger et al. (1989). Output values were reduced for targets following phonetically related primes, relative to unrelated primes. If the subsequently presented target is phonetically similar to the prime, the residual activation left by the prime will inhibit processing of the target, because of the inhibitory connections among units within a level. Thus, the simple phenomenon of residual activation, combined with the mechanism of within-level inhibition, gives rise to inhibitory phonetic priming.

\section{GENERAL DISCUSSION}

Despite marked differences in the stimulus sets and experimental paradigms employed in Goldinger et al. (1989) and the present study, the patterns of results are remarkably similar, demonstrating that the observed ef- fects of short-term, inhibitory phonetic priming are robust across items and tasks. We also demonstrated that PARSYN, a connectionist instantiation of the NAM, successfully simulates the observed effects of inhibition priming. Together, our behavioral and simulation results support the basic claims of any of a class of current activationcompetition models. Moreover, the phonetic priming effect suggests that neighborhoods of phonetic patterns are activated in memory on the basis of stimulus input and that multiple activation of competing phonetic patterns slows processing.

The present investigation contributes to a growing body of literature on form-based priming in spoken word recognition (Goldinger et al., 1989; Goldinger et al., 1992; Radeau et al., 1989; Radeau, Morais, \& Segui, 1995; Slowiaczek \& Hamburger, 1992; Slowiaczek et al., 1987). A facile summary of the results of this literature is, at present, not possible. Various researchers (Monsell \& Hirsh, 1998; Slowiaczek \& Hamburger, 1992; Slowiaczek et al., 1987) have reported facilitative--rather than inhibitory priming effects when spoken primes and targets share segments (in contrast to the stimuli used here, which had no positional overlap). It is now clear that at least some of these effects are primarily strategic (see Goldinger et al., 1992), arising from subjects' expectancies of similarity relations between the prime and the target. In contrast, the type of inhibitory phonetic priming observed in the present study seems relatively impervious to subject strategies, presumably because of the subtlety of the similarity relations. In short, some facilitative priming effects may arise because segmental overlap induces subjects to generate expectancies about the target on the basis of the prime, thus enhancing processing of the target.

A second possible reason for the difference in priming effects as a function of overlap is that facilitation effects may actually arise from residual activation in the processing system. For example, Gagnon et al. (2000) demonstrated facilitation when primes and targets shared segments, even when strategic processing was unlikely. Thus, on the basis of the available evidence, it is important that the same model that produces inhibition effects in the absence of segmental overlap can produce nonstrategic facilitation effects for overlapping primes and targets. Simulations of PARSYN using stimuli from Gagnon et al. demonstrate that residual activation from identical overlapping segments does, indeed, produce facilitative priming. Allophone and pattern units in PARSYN benefit from previously presented identical segments, thus producing an overall net facilitation effect. Thus, the existence of both facilitative and inhibitory priming effects is not inconsistent with the present processing model.

Monsell and Hirsh (1998) also attempt to clarify the seemingly disparate previous findings on competitive and facilitative effects in form-based priming. In their review of the literature, they conclude that "it requires the eye of faith to see inhibitory priming as the dominant pattern emerging from experiments with a short lag be- 
tween prime and probe" (p. 1,499). Thus, in an effort to place effects of inhibitory priming on a firmer empirical footing, the authors conducted a series of lexical decision experiments examining form-based priming for spoken words, in which they varied the lag between prime and target. Monsell and Hirsh also manipulated lexicality, length in syllables, and position of overlap (beginning vs. ending). The subjects in their experiments made lexical decisions (word vs. nonword) to both the primes and the targets.

Despite the numerous manipulations examined by the authors, the results of only a handful of conditions are easily interpreted. The reasons for the difficulties in assessing Monsell and Hirsh's (1998) data stem primarily from their use of the lexical decision task. First, handedness was confounded with response type, thus making interpretation of priming effects (or lack thereof) for nonword primes and targets difficult, given the additional time required to respond to nonwords. (Nonword responses were approximately 50 to $100 \mathrm{msec}$ longer than the word responses.) Second, requiring subjects to respond negatively to nonword primes in the lexical decision task may result in inhibition of nonword patterns, potentially reducing their ability to act as effective primes (see P. A. Luce \& Lyons, 1998). Finally, the effects of form-based priming on nonwords that are similar to real words (such as those employed by Monsell and Hirsh) may be overshadowed by competition effects from realword neighbors. In short, interpretation of the results from conditions containing either nonword primes or targets is problematic.

The findings from the remaining conditions involving only word primes and targets demonstrated a mixed pattern of inhibition and facilitation for monosyllabic stimuli (just as in previous short-lag experiments; see Hamburger \& Slowiaczek, 1996). For both mono- and multisyllabic primes and targets sharing initial segments, Monsell and Hirsh (1998) observed inhibitory priming effects, even at long lags. However, for monosyllabic stimuli sharing final segments, facilitative priming effects occurred.

The present finding that inhibitory priming dissipates over a brief interval appears to contradict some of the long-lasting competitive effects demonstrated by Monsell and Hirsh (1998). However, their long-term priming data may have little relevance for the short-term, transient priming observed in this (and other) studies, primarily because the long-term competitive effect may be strategic. Monsell and Hirsh observed both facilitative and competitive priming in the same experiments. Curiously, the authors provide an activation-based, cohort account of the competitive effects while, at the same time, attributing the facilitative effects to "conscious detection of rhymes at long intervals" on the basis of "some separate and explicit representation of phonological sequence" (p. 1,513). One likely alternative is that conscious detection of overlap operates in both conditions with dif- ferent consequences. Given initial overlap, listeners may reactivate the prime while the target unfolds, creating competition (see Ratcliff, Allbritton, \& McKoon, 1997). Such an interpretation does not require postulating a long-lasting shift in baseline activation or rate of activation growth. Strategic recoliection (or reactivation) of the prime on the basis of overlapping stimulus input is sufficient. For primes and target sharing final segments, listeners most likely generate a single dominant hypothesis regarding the identity of the stimulus by the time the overlap is detected (Marslen-Wilson \& Welsh, 1978), thus attenuating or eliminating strategy-based competitive effects. As Monsell and Hirsh themselves suggest, strategic detection of overlapping segments in this condition may speed lexical decision times by some unspecified "conscious" process. Indeed, Monsell and Hirsh's experiments meet all of the criteria previously established for strategybased priming: (1) a high proportion of overlapping items in relatively small stimulus sets (Goldinger, 1998; Goldinger et al., 1992), (2) subjects' own admissions that they noticed the overlapping items (Monsell \& Hirsh, 1998, p. 1,511), and (3) long-term form-based (nonidentity) priming arising from a single exposure to the prime, with little diminution of the effect over time. (Monsell and Hirsh report, at most, a 7-msec decline in the "priming" effect over a matter of minutes; see Posner \& Snyder, 1975a, 1975b.) ${ }^{1}$

Monsell and Hirsh (1998) also raise a number of questions regarding our previous (and, by extension, present) interpretation of the phonetic priming effect, one of which is addressed by the present experiments. They ask why no effects of phonetic priming were observed in Goldinger et al. (1992) at a 50-msec ISI with stimuli presented in the clear. As we have demonstrated, such inhibitory effects are indeed present when phonetic similarity is carefully manipulated, and these effects quickly dissipate over a longer ISI. Monsell and Hirsh also argue that short-lag inhibitory priming requires primes and targets sharing initial segments, but the present data show that their conclusion is unfounded.

Monsell and Hirsh (1998) also ask why strategic effects do not also operate in the clear. Again, the answer is that they do, as is demonstrated by Goldinger (1999). Finally, the authors express surprise that the priming observed by Goldinger et al. (1989; Goldinger et al., 1992) is based on prime-target pairs without overlapping segments. Careful reading of those studies reveals, however, that this choice of primes was deliberate: According to Goldinger et al. (1989), "The restriction against overlapping phonemes was imposed in order to prevent subjects from generating response strategies based on repeated overlap between prime-target pairs" (p. 504). Thus, we anticipated Monsell and Hirsh's insights (see p. 1,499) 9 years in advance of their claim that inhibitory priming may be masked by strategic effects induced by overlapping items. Attention to previously published work would have 
dispelled the apparent bewilderment embodied in their question, "Why does a competitor effect reveal itself reliably only when proximity of the prime and probe is so low as to be intuitively nonobvious (veer and bull) but not when they have several phonemes or a syllable in common ...?"' (p. 1,499).

All told, Monsell and Hirsh's (1998) arguments regarding short-term competitive priming are contradicted by the evidence in this and previous studies. Moreover, owing to methodological shortcomings, their results do little to clarify previously reported effects of facilitative and competitive priming. On the other hand, the present findings provide clear support for the existence of shortterm inhibitory effects based on momentary changes in activation levels of lexical neighbors. Furthermore, our results are most consistent with the kinds of processing architectures proposed in TRACE, NAM, or PARSYN, rather than with a strict cohort-based view of the recognition process (as espoused by Monsell and Hirsh).

In summary, we have demonstrated that form-based inhibition priming in spoken word recognition is a robust finding: It does not depend on specific stimuli, stimulus degradation, or experimental paradigm. Moreover, we have shown that phonetically related primes have demonstrable effects on processing times for target words. Finally, we successfully simulated our results by using PARSYN, a connectionist instantiation of the NAM. We believe that our empirical and simulation results lend strong support to a general class of models of spoken word recognition that places special emphasis on the roles of activation and competition in the perception of spoken stimuli.

\section{REFERENCES}

Auer, E. T., \& LuCE, P. A. (2000). PARSYN: A processing model of neighborhood activation and phonotactics in spoken word recognition. Manuscript in preparation.

Cluff, M. S., \& LuCE, P. A. (1990). Similarity neighborhoods of spoken two-syllable words: Retroactive effects on multiple activation. Journal of Experimental Psychology: Human Perception \& Performance, 16, 551-563.

Collins, A., \& LofTus, E. (1975). A spreading activation theory of semantic processing. Psychological Review, 82, 407-428.

Gagnon, D., Palmer, N., \& SAwusch, J. (2000). The segmental representation of spoken words. Manuscript submitted for publication.

Goldinger, S. D. (1998). Signal detection comparisons of phonemic and phonetic priming: The flexible-bias problem. Perception \& Psychophysics, 60, 952-965.

Goldinger, S. D. (1999). Only the Shadower knows: Comment on Hamburger and Slowiaczek (1996). Psychonomic Bulletin \& Review, 6, 347-351.

Goldinger, S. D., Luce, P. A., \& Pisoni, D. B. (1989). Priming lexical neighbors of spoken words: Effects of competition and inhibition. Journal of Memory \& Language, 28, 501-518.

Goldinger, S. D., Luce, P. A., Pisoni, D. B., \& Marcario, J. K. (1992). Form-based priming in spoken word recognition: The roles of competition and bias. Journal of Experimental Psychology: Learning, Memory, \& Cognition, 18, $1211-1238$.

Hamburger, M., \& Slowiaczek, L. M. (1996). Phonological priming reflects lexical competition. Psychonomic Bulletin \& Review, 3, 520525 .
Kučera, H., \& Francis. W. N. (1967). Computational analysis of present-day American English. Providence, RI: Brown University Press.

LUCE, P. A. (1986). Neighborhoods of words in the mental lexicon (Research on Speech Perception, Tech. Rep. No. 6). Bloomington: Indiana University, Department of Psychology, Speech Research Laboratory.

LUCE, P. A., \& LYONS, E. A. (1998). Specificity of memory representations for spoken words. Memory \& Cognition, 26, 708-715.

LuCE. P. A., \& Pisoni, D. B. (1998). Recognizing spoken words: The neighborhood activation model. Ear \& Hearing, 19, 1-36.

Luce, P. A., Pisoni, D. B., \& Goldinger, S. D. (1990). Similarity neighborhoods of spoken words. In G.T. M. Altmann (Ed.), Cognitive models of speech processing: Psycholinguistic and computational perspectives. Cambridge, MA: MIT Press.

LUCE, R. D. (1959). Individual choice behavior. New York: Wiley.

Marsi.en-Wilson, W. D. (1989). Access and integration: Projecting sound onto meaning. In W. D. Marslen-Wilson (Ed.), Lexical access and representation ( $\mathrm{pp}$. 3-24). Cambridge, MA: Bradford Books.

MARSLEN-WILSON, W. D., \& Welsh, A. (1978). Processing interactions and lexical access during word recognition in continuous speech. Cognitive Psychology, 10, 29-63.

McClelland, J. L., \& Elman. J. L. (1986). The TRACE model of speech perception. Cognitive Psvchologv, 18, 1-86.

MCClelland, J. L.. \& Rumel.hart, D. E. (1981). An interactive activation model of context effects in letter perception: Pt. 1. An account of the basic findings. Psychological Review, 88, 375-407.

MCQueen, J., Norris, D.. \& CutLer, A. (1994). Competition in word recognition: Spotting words in other words. Journal of Experimental Psychology: Learning, Memory, \& Cognition, 20, 621-638.

Monsell, S., \& HiRsh, K. W. (1998). Competitor priming in spoken word recognition. Journal of Experimental Psychology: Learning. Memory, \& Cognition, 24, 1495-1520.

MorTON. J. (1969). Interaction of information in word recognition. Psychological Review, 76, 165-178.

NORRIS, D. (1994). Shortlist: A connectionist model of continuous speech recognition. Cognition, 52, 189-234.

Norris, D.. MCQueen, J. M.. \& CuTler, A. (1995). Competition and segmentation in spoken-word recognition. Journal of Experimental Psychology: Learning, Memory, \& Cognition, 21, 1209-1228.

Nusbaum, H. C., Pisoni, D. B., \& Davis, C. K. (1984). Sizing up the Hoosier mental lexicon: Measuring the familiarity of 20.000 nords (Research on Speech Perception Progress Report No. 10). Bloomington: Indiana University, Psychology Department. Speech Research Laboratory.

Posner, M. I., \& Snyder, C. R. R. (1975a). Attention and cognitive control. In R. Solso (Ed.), Information processing and cognition: The Loyola symposium (pp. 55-85). Potomac, MD: Erlbaum.

Posner, M. I., \& SNyder, C. R. R. (1975b). Facilitation and inhibition in the processing of signals. In P. M. A. Rabbit \& S. Dornic (Eds.), Attention and performance $V$ (pp. 669-682). New York: Academic Press.

Radeau, M., Morais, J., \& Dewier, A. (1989). Phonological priming in spoken word recognition: Task effects. Memory \& Cognition, 17. 525-535.

Radeau, M., Morais, J., \& Segul. J. (1995). Phonological priming between monosyllabic spoken words. Journal of Experimental Psychology: Human Perception \& Performance, 21, 1297-1311.

RatClifF, R., AllbritTon. D., \& MCKoON. G. (1997). Bias in auditory priming. Journal of Experimental Psychologv: Learning. Memory; \& Cognition, 23, 143-152.

Slowiaczek, L. M., \& Hamburger, M. (1992). Prelexical facilitation and lexical interference in auditory word recognition. Journal of Experimental Psychology: Learning, Memory, \& Cognition, 18, 1239-1250.

Slowiaczek, L. M., Nusbaum H. C.. \& Pisoni, D. B. (1987). Phonological priming in auditory word recognition. Journal of Experimental Psychology: Learning. Memory, \& Cognition, 13, 64-75.

Vitevitch, M. S., \& LuCE, P. A. (1998). When words compete: Levels of processing in spoken word perception. Psychological Science, 9. 325-329.

Vitevitch, M. S., \& LuCE. P. A. (1999). Probabilistic phonotactics and 
neighborhood activation in spoken word recognition. Journal of Memory \& Language, 40, 374-408.

Webster's Seventh Collegiate Dictionary (1967). Los Angeles: Library Reproduction Service.

\section{NOTE}

1. The authors argue against a bias account of their competitive priming effects on the basis of data from an experiment in which they in- creased the lags between prime and target (Experiment 4), but subjects may still be able to employ overlap strategically in this condition (see Ratcliff et al., 1997). Once overlapping targets begin appearing at longer lags, there is no reason to expect that subjects will have forgotten the previous presentations of primes and, thus, be ignorant of the unfolding evidence that some of the stimuli overlap in systematic ways, especially when stimulus sets with such high proportions of overlapping stimuli are used. Unfortunately, increasing lag does little to demonstrate convincingly that strategic effects are inoperative.

\section{APPENDIX}

\begin{tabular}{|c|c|c|c|c|c|}
\hline Target & $\begin{array}{l}\text { Higher Frequency } \\
\text { Related Prime }\end{array}$ & Unrelated Prime & Target & $\begin{array}{l}\text { Lower Frequency } \\
\text { Related Prime }\end{array}$ & Unrelated Prime \\
\hline thin & them & suit & gong & shun & weed \\
\hline van & thing & sake & hum & pawn & sage \\
\hline jail & share & voice & hatch & peg & chum \\
\hline fame & then & such & booth & hoof & leak \\
\hline sung & join & peace & huff & poise & cheat \\
\hline cheap & set & whose & ken & tame & roach \\
\hline cease & jazz & cool & hut & poop & cheese \\
\hline pill & hair & loose & gum & churn & leach \\
\hline heap & pit & June & jar & shawl & niece \\
\hline surge & choice & mean & palm & vine & reap \\
\hline rug & walk & team & mess & hash & coil \\
\hline par & haul & sheet & peas & hiss & soup \\
\hline $\operatorname{tar}$ & pall & reach & gear & shell & loop \\
\hline heal & peer & choice & height & pop & chore \\
\hline peer & hell & shot & foam & bun & cheek \\
\hline doll & fire & lease & pope & hoot & seal \\
\hline tune & come & week & teach & kiss & womb \\
\hline pearl & hung & seek & wire & yell & tape \\
\hline mock & hope & keen & ride & watt & coal \\
\hline wreath & laugh & coach & choose & shove & veal \\
\hline thief & faith & soon & $\sin$ & gem & coop \\
\hline pet & hip & bone & phone & thumb & chic \\
\hline lure & full & keep & soil & chore & peach \\
\hline job & side & peace & lobe & thud & geese \\
\hline rule & wore & deep & suck & chirp & peal \\
\hline path & his & such & siege & chess & hoop \\
\hline sheep & sit & cool & sought & chop & kneel \\
\hline $\operatorname{gin}$ & seem & voice & hoot & pup & cheese \\
\hline rob & wide & chief & thumb & fawn & cheat \\
\hline lodge & wash & team & rack & wed & cane \\
\hline rail & wear & coach & shed & sage & coil \\
\hline vale & fair & chose & bake & vet & shore \\
\hline pine & calm & search & cone & tomb & leach \\
\hline pit & heap & soul & pin & hem & soup \\
\hline map & net & use & cape & pet & roar \\
\hline hull & pore & seed & noon & hum & cheap \\
\hline hell & pair & choice & rice & lodge & coal \\
\hline tear & pill & load & folk & thug & gene \\
\hline wool & roar & beach & bus & verge & seal \\
\hline merge & thus & beam & tongue & coin & weep \\
\hline cage & guess & room & fool & bore & neat \\
\hline chef & save & coin & wear & rail & coop \\
\hline pig & head & soon & fun & thong & chic \\
\hline pop & height & lean & tide & $c o b$ & wail \\
\hline gore & cool & leap & wet & rip & tool \\
\hline rouge & wood & keep & theme & fine & goose \\
\hline
\end{tabular}

